\section{AB032. Posterior mediastinal paravertebral Mullerian cyst resected by uniportal video- assisted thoracoscopic surgery: case report and review of literature}

Ping-Chung Tsai ${ }^{1}$, Yi-Chen $\mathrm{Yeh}^{2}$, Po-Kuei $\mathrm{Hsu}^{1}$, YuChung $\mathrm{Wu}^{1}$

${ }^{1}$ Departments of Surgery, ${ }^{2}$ Department of Pathology, Division of Thoracic Surgery, Taipei Veterans General Hospital, Taipei, Taiwan

Abstract: Since Hattori first described a mediastinal cyst with a feature characteristic of a Mullerian cyst in 2005 , there has been several similar cases reported in the literature. Frequently misclassified as bronchogenic cyst, it is recognized that mediastinal Mullerian cysts is a unique type of mediastinal cyst with biologically distinctive characteristics. A 44-year-old woman, who had past medical history of hysterectomy for uterine leiomyomas and adenomyosis were incidentally found to have abnormal findings on chest roentgenogram. Subsequent chest computed tomography (CT) revealed a homogenous soft tissue lesion at left side paravertebral area, which was very close to descending aorta. Under the impression of bronchogenic cyst or cystic schwannoma, she underwent left side uniportal video-assisted thoracoscopic surgery
(VATS). A cyst-like mass was identified paravertebrally, without evidence of invasion to the descending aorta. Pathologic examination revealed $1.2 \times 1.2 \times 0.4 \mathrm{~cm}$ cystic lesion with a single layer of columnar epithelial lining. The columnar-lining epithelial cells stained positive with immunohistochemical stains for CK, CK7, estrogen receptor (ER), progesterone receptor (PR), paired box gene 8 (PAX8), and negative for CK20, TTF-1, and calretinin. The overall histologic and immunohistochemical findings were compatible with Mullerian cysts. The origin of a Mullerian cyst in the mediastinum is unclear. Clinical features include generally in woman aged 40 to 60 years, frequently with obesity and gynecologic history, typically presented with cough, pain or asymptomatic, and always posterior mediastinal paravertebral location, occurring between T3-T8 vertebrae. The behavior of mediastinal Mullerian cysts appears benign and there are no present reports of recurrence, it should be considered in the differential diagnosis of posterior mediastinal paravertebral cysts.

Keywords: Posterior mediastinal mass; Mullerian cyst; thoracoscopic surgery; fifty females

doi: $10.21037 /$ jtd.2017.s032

Cite this abstract as: Tsai PC, Yeh YC, Hsu PK, Wu YC. Posterior mediastinal paravertebral Mullerian cyst resected by uniportal video-assisted thoracoscopic surgery: case report and review of literature. J Thorac Dis 2017;9(Suppl 14):AB032. doi: $10.21037 /$ jtd.2017.s032 